\title{
Development and Validation of a New Blade Element Momentum Skewed-Wake Model within AeroDyn
}

\author{
Andrew Ning \\ Brigham Young University, aning@byu.edu \\ Greg Hayman \\ National Renewable Energy Laboratory \\ Rick Damiani \\ National Renewable Energy Laboratory \\ Jason Jonkman \\ National Renewable Energy Laboratory
}

Follow this and additional works at: https://scholarsarchive.byu.edu/facpub

Part of the Mechanical Engineering Commons

\section{Original Publication Citation}

Ning, A., Hayman, G., Damiani, R., and Jonkman, J., “Development and Validation of a New Blade Element Momentum Skewed-Wake Model within AeroDyn," 33rd ASME Wind Energy Symposium, Kissimmee, FL, Jan. 2015. doi:10.2514/6.2015-0215

\section{BYU ScholarsArchive Citation}

Ning, Andrew; Hayman, Greg; Damiani, Rick; and Jonkman, Jason, "Development and Validation of a New Blade Element Momentum Skewed-Wake Model within AeroDyn" (2015). Faculty Publications. 1725. https://scholarsarchive.byu.edu/facpub/1725 


\title{
Development and Validation of a New Blade Element Momentum Skewed-Wake Model within AeroDyn
}

\author{
S. Andrew Ning* \\ Brigham Young University, Provo, UT \\ Greg Hayman ${ }^{\dagger}$, Rick Damiani ${ }^{\ddagger}$, and Jason Jonkman ${ }^{\ddagger}$ \\ National Renewable Energy Laboratory, Golden, CO
}

\begin{abstract}
Blade element momentum methods, though conceptually simple, are highly useful for analyzing wind turbines aerodynamics and are widely used in many design and analysis applications. A new version of AeroDyn is being developed to take advantage of new robust solution methodologies, conform to a new modularization framework for National Renewable Energy Laboratory's FAST, utilize advanced skewed-wake analysis methods, fix limitations with previous implementations, and to enable modeling of highly flexible and nonstraight blades. This paper reviews blade element momentum theory and several of the options available for analyzing skewed inflow. AeroDyn implementation details are described for the benefit of users and developers. These new options are compared to solutions from the previous version of AeroDyn and to experimental data. Finally, recommendations are given on how one might select from the various available solution approaches.
\end{abstract}

\section{Nomenclature}

$B \quad$ number of blades [parameter]

$C_{Q} \quad$ local torque coefficient

$C_{T} \quad$ local thrust coefficient

$F \quad$ hub/tip correction factor

$M_{z}^{\prime} \quad$ moment per unit length about the airfoil coordinate system z-direction

$R \quad$ rotor tip radius [input]

$R_{\text {hub }}$ rotor hub radius [parameter]

Re Reynolds number

$V \quad$ velocity

$W \quad$ inflow velocity [output]

$X^{\prime} \quad$ force per unit length in the airfoil coordinate system x-direction

$Y^{\prime} \quad$ force per unit length in the airfoil coordinate system y-direction

$\Phi \quad$ precone angle

$\Theta \quad$ tilt angle

$\alpha \quad$ angle of attack

$\chi \quad$ wake-skew angle

$\chi_{0} \quad$ inflow-skew angle [input]

$\gamma \quad$ yaw angle

$\kappa \quad$ axial parameter

$\kappa^{\prime} \quad$ tangential parameter

$\mu \quad$ dynamic viscosity [parameter]

*Assistant Professor, Mechanical Engineering Department, AIAA Member

†Software Engineer, National Wind Technology Center

¥Senior Engineer, National Wind Technology Center, AIAA Member 
$\phi \quad$ local inflow angle [output, state for uncoupled methods]

$\psi \quad$ azimuth angle [input]

$\rho \quad$ fluid density [parameter]

$\sigma^{\prime} \quad$ local solidity $(B c /(2 \pi r))$

$\theta \quad$ twist angle (geometric + structural) [input]

$\theta_{p} \quad$ pitch angle [input]

$a \quad$ axial induction factor [state for coupled methods]

$a^{\prime} \quad$ tangential induction factor [state for coupled methods]

$c \quad$ chord [parameter]

$c_{d} \quad$ drag coefficient

$c_{l} \quad$ lift coefficient

$r \quad$ local radial position [input]

\section{Introduction}

Blade element momentum (BEM) theory continues to be widely used for wind turbine applications such as initial aerodynamic analysis, conceptual design, loads and stability analysis, and controls design. Recent advances in deriving a robust solution algorithm for BEM theory [1] and establishing a new modularization framework for FAST [2] have prompted development of a new version of AeroDyn. One aspect of that development is in the treatment of skewed wakes (for yawed or tilted flows). Skewed wakes have renewed interest in the wind engineering community as research on wind plant underproduction has led to studies on wake steering using control of the yaw error. Of course, even without this type of control, skewed wakes are always prevalent for turbines operating under stochastic wind conditions. The previous version of AeroDyn used only a simple skewed wake correction method. Several methodologies for analyzing skewed wakes exist, and it is unclear which formulations are most appropriate and under which conditions. This paper will not attempt to definitively answer that question, but will describe some of the options that are available in the new version of AeroDyn and show comparisons to experimental measurement campaigns. The description in this paper also serves as a review of some of the methodologies used to assess skewed-wake BEM flows.

Two main methods are described in this paper, each with several variations. The first class of methods will be called correction methods. These methods provide corrections to computed induction factors, power coefficients, or other quantities. Several authors have proposed different varieties of the classic Glauert's [3] first harmonic correction for the inflow. These approximations derive from rotorcraft theory and assume a longitudinal or both a longitudinal and a lateral variation of the inflow across the rotor plane. More complex theories, such as those based on vortex and flow expansion [4-6] and those based on acceleration potential methods as in $[7,8]$, have also been used to arrive at inflow corrections for yawed conditions. These corrective equations typically contain sine and cosine terms of the azimuth angle and a linear dependence on the radial distance from the hub (e.g., $[7,9,10])$. The robust solution method in [1] can easily be extended to handle these methods.

The second class of approaches will be called coupled methods. In these methods, the inclusion of skewedwake effects is integral to the derivation, and the calculation of the axial and tangential induction factors are not separable. The coupled methods cannot be solved using the new robust solution algorithm, and must use traditional two-dimensional root-finding approaches.

Another important aspect of analyzing a rotor operating in skew is the unsteady aerodynamic behavior of the airfoil sections. However, this physics is handled in a separate module of AeroDyn and is not applied or otherwise discussed here.

The local airfoil coordinate system used in the derivation of both BEM theories is shown in Fig. 1. The $\mathrm{z}$-direction is directed along the blade, the $\mathrm{y}$-direction opposite to the rotational velocity, and the $\mathrm{x}$-direction given by the right-hand rule (nominally in the downwind direction). This coordinate system is equally valid for downwind turbines, but in both cases, assumes that the rotor is rotating clockwise when viewed from upwind. Because the blade may be curved (whether due to a nonstraight design or deflection), the coordinate system is local to each section along the blade (Fig. 1a). Swept blades can also be handled, but it is assumed that sweep is accomplished through shearing, rather than rotation, so that the local airfoils and local coordinate systems are still defined relative to the unswept rotation direction (Fig. 1b). 


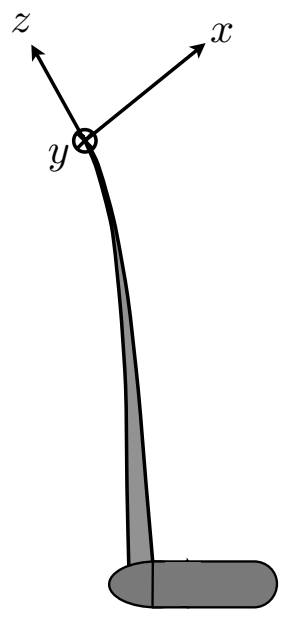

(a) Side view of curved blade. Coordinate system is normal to curvature.

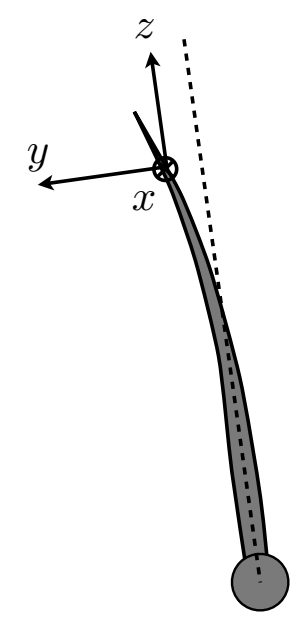

(b) Front view of swept blade. Sweep is accomplished through shearing, so coordinate system stays parallel to sweep of blade root.

Figure 1: Definition of local airfoil coordinate system.

\section{Classical BEM Theory with a Guaranteed Solution Algorithm}

This section reviews the procedure for solving the BEM equations using the guaranteed solution method of Ning [1]. This procedure is based around forming a one-dimensional residual equation as a function of the local inflow angle $(\mathcal{R}(\phi))$. The definition of the inflow angles and velocity components is seen in Fig. 2.

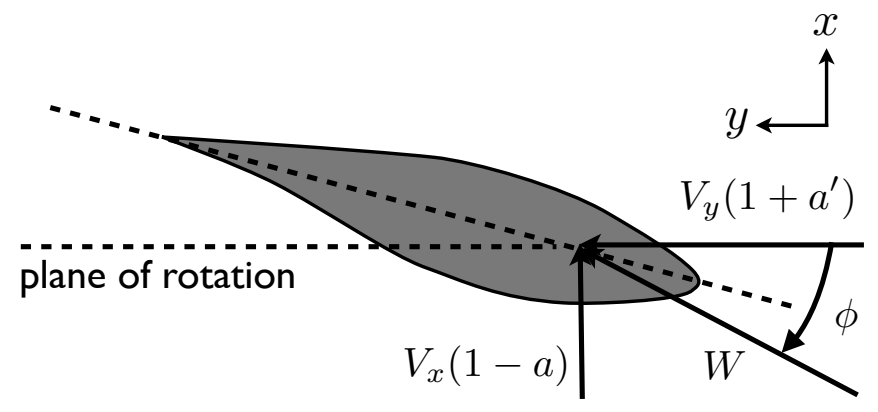

Figure 2: Definition of velocity components and inflow parameters for a local rotating blade section looking toward the root from the tip.

First, sectional theory is used to compute the force coefficients (Eqs. (1)-(7)). In the simplest case, the velocity components are given by $V_{x}=V_{\infty}$ and $V_{y}=\Omega r$, but in the general case, the velocity components must also account for shear, turbulence, blade motion, and blade orientation. Generally, this is accomplished by using a direction cosine matrix to define the 3D orientation and to take relative (wind/structural) velocities. The Reynolds number can include the induction factors, but that is typically unnecessary because relevant changes in Reynolds number are usually of a much higher order of magnitude than can be caused by changes in induction factor. The theory permits induction factors to be included in the Reynolds number calculation through an extra iteration loop, but in practice this is rarely useful [1]. The functions $f_{L}$ and $f_{D}$ used in Eqs. (4) and (5) are often lookup tables from precomputed airfoil data, preferably with continuously differentiable splines. In Eqs. (6) and (7) the drag coefficient is omitted in some implementations, and is optional in AeroDyn. Note that the positive direction for $c_{y}$ is opposite the $y$ vector by convention. 


$$
\begin{aligned}
\alpha & =\phi-\left(\theta+\theta_{p}\right) \\
W_{\infty} & =\sqrt{V_{x}^{2}+V_{y}^{2}} \\
R e & =\frac{\rho W_{\infty} c}{\mu} \\
c_{l} & =f_{L}(\alpha, R e) \\
c_{d} & =f_{D}(\alpha, R e) \\
c_{x} & =c_{l} \cos \phi+c_{d} \sin \phi \\
c_{y} & =c_{l} \sin \phi-c_{d} \cos \phi
\end{aligned}
$$

Next, the results of blade element theory are combined with those of momentum theory in order to compute the induction factors. In the following, it is assumed that the empirical region is estimated using Glauert's correction with Buhl's modification [11]. First, two relevant nondimensional parameters are computed as

$$
\begin{aligned}
\kappa & =\frac{\sigma^{\prime} c_{x}}{4 F \sin ^{2} \phi} \\
\kappa^{\prime} & =\frac{\sigma^{\prime} c_{y}}{4 F \sin \phi \cos \phi}
\end{aligned}
$$

where $F$ is a hub/tip loss correction. AeroDyn uses Prandtl's correction

$$
\begin{aligned}
f_{\text {tip }} & =\frac{B}{2}\left(\frac{R-r}{r|\sin \phi|}\right) \\
F_{\text {tip }} & =\frac{2}{\pi} \arccos \left(\exp \left(-f_{\text {tip }}\right)\right) \\
f_{\text {hub }} & =\frac{B}{2}\left(\frac{r-R_{\text {hub }}}{R_{\text {hub }}|\sin \phi|}\right) \\
F_{\text {hub }} & =\frac{2}{\pi} \arccos \left(\exp \left(-f_{\text {hub }}\right)\right) \\
F & =F_{\text {tip }} F_{\text {hub }}
\end{aligned}
$$

Different equations must be used depending on the solution region. If $\phi>0$ and $\kappa \leq 2 / 3$ then the solution falls in the momentum region where

$$
a=\frac{\kappa}{1+\kappa}
$$

Alternatively, if $\phi>0$ and $\kappa>2 / 3$ then the solution falls in the empirical region.

$$
a=\frac{\gamma_{1}-\sqrt{\gamma_{2}}}{\gamma_{3}}
$$

where

$$
\gamma_{1} \equiv 2 F \kappa-\left(\frac{10}{9}-F\right), \quad \gamma_{2} \equiv 2 F \kappa-F\left(\frac{4}{3}-F\right), \quad \gamma_{3} \equiv 2 F \kappa-\left(\frac{25}{9}-2 F\right)
$$

If the denominator in Eq. (17) is exactly zero (i.e., $\gamma_{3}=0$ ), then the numerator is also exactly zero. However, the expression can still be evaluated using L'Hôpital's rule and can be shown to be equal to

$$
a \stackrel{\gamma_{3} \rightarrow 0}{\longrightarrow} 1-\frac{1}{2 \sqrt{\gamma_{2}}}
$$

Otherwise if $\phi<0$ and $\kappa>1$, then the solution lies in the propeller brake region.

$$
a=\frac{\kappa}{\kappa-1}
$$

$$
4 \text { of } 18
$$


If $\phi<0$ and $\kappa \leq 1$ then this value of $\phi$ cannot possibly be a solution to the BEM equations and so we can set $a$ to any value for which the residual is guaranteed to be nonzero. For this formulation this holds by simply setting $a=0$. Finally, the tangential induction factor is given as

$$
a^{\prime}=\frac{\kappa^{\prime}}{1-\kappa^{\prime}}
$$

With the induction factors computed, the residual can be calculated as

$$
\mathcal{R}(\phi)=\frac{\sin \phi}{1-a}-\frac{V_{x}}{V_{y}} \frac{\cos \phi}{\left(1+a^{\prime}\right)}
$$

With equations Eqs. (1)-(22) comprising the evaluation of the residual $\mathcal{R}(\phi)$, the solution to the residual equation can be found using a root finding method such as Brent's method [12] as outlined in Algorithm 1. The solution, $\phi^{*}$, is plugged back into Eqs. (1)-(7) in order to compute the loads. For the loads calculation, the drag coefficient should always be included in Eqs. (6) and (7), and the induction factors should be included in evaluating $W$ for use in dimensionalizing the forces. The normal and tangential loads per unit length along the blade are

$$
\begin{aligned}
W & =\sqrt{\left(V_{x}(1-a)\right)^{2}+\left(V_{y}\left(1+a^{\prime}\right)\right)^{2}} \\
q & =\frac{1}{2} \rho W^{2} \\
X^{\prime} & =c_{x} q c \\
Y^{\prime} & =-c_{y} q c \\
M_{Z}^{\prime} & =q c^{2} c_{m}
\end{aligned}
$$

The integration of these loads along the blade for thrust and torque must account for the curvature, coning, and azimuthal orientation of the blades.

The BEM equations breakdown if one of the velocity components is exactly zero (i.e., $V_{x}=0$ or $V_{y}=0$ in Fig. 2). This can occur, for example, in parked conditions or near yaw angles of $90^{\circ}$. For these cases, AeroDyn sets both induction factors to zero, and computes the loads in the same manner as described in Eqs. (23)-(27).

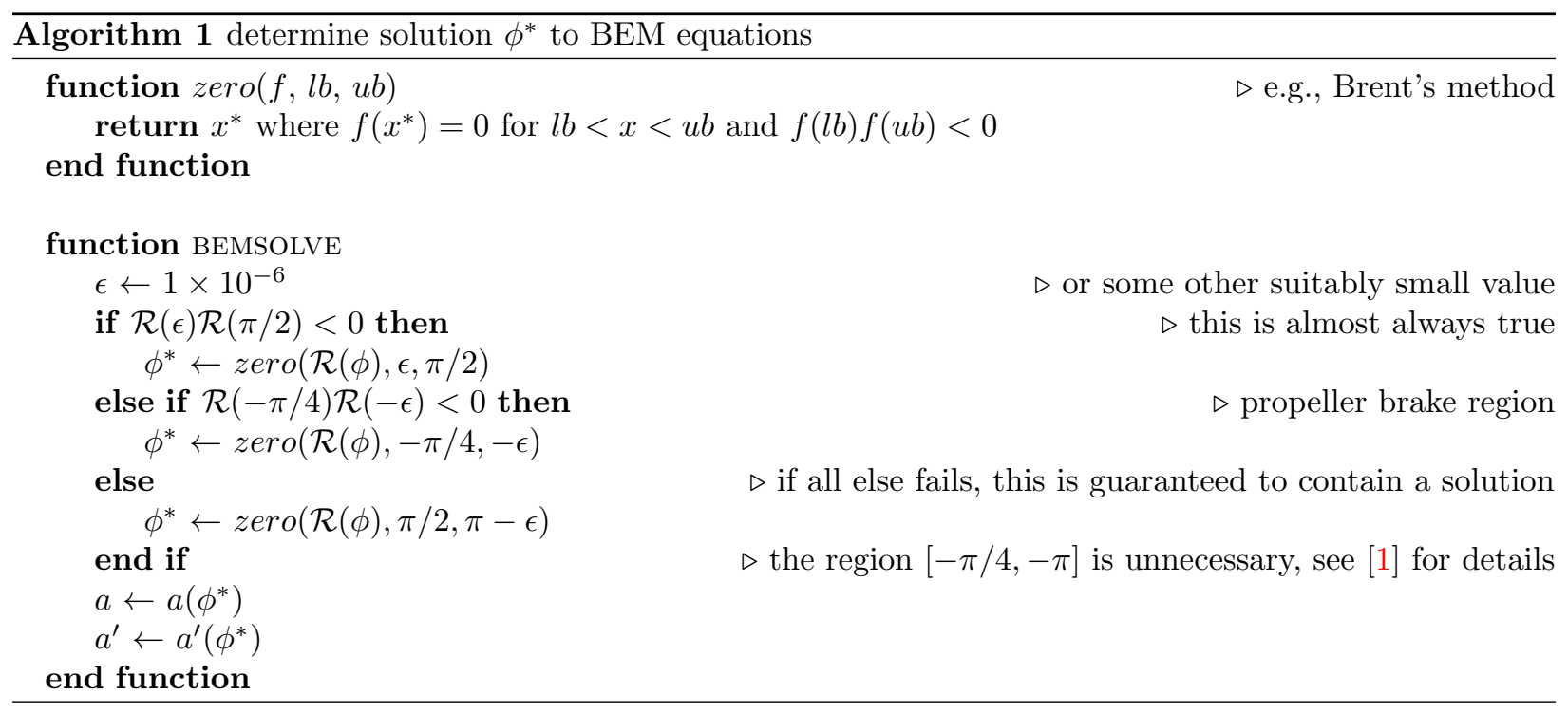

\section{Corrections Methods Compatible with Basic BEM Implementations}

There are multiple approaches to applying skewed-wake corrections to the basic BEM formulation. From the blade element perspective, the only change needed to account for skew is in computing the in-plane 
velocity components ( $V_{x}$ and $V_{y}$ in Eqs. (2) and (23)). As an example, assuming conventional static rotor geometry operating with yaw and tilt under uniform flow, the wind components can be computed as

$$
\begin{aligned}
& V_{x}=V_{\infty}((\cos \gamma \sin \Theta \cos \psi+\sin \gamma \sin \psi) \sin \Phi+\cos \gamma \cos \Theta \cos \Phi) \\
& V_{y}=V_{\infty}(\cos \gamma \sin \Theta \sin \psi-\sin \gamma \cos \psi)+\Omega r \cos \Phi
\end{aligned}
$$

where the definition of the angles are shown in Fig. 3. Positive precone angles $(\Phi)$ tilt the blades upwind. For curved blades and dynamic motion the equations are more complex, but all cases simply involve resolving the velocity components into the local airfoil frame.

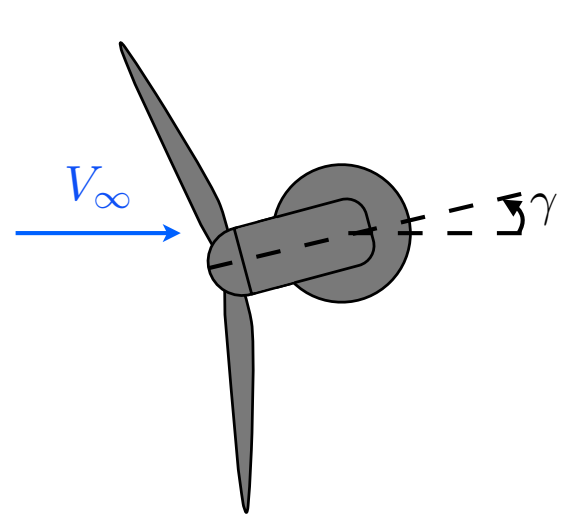

yaw

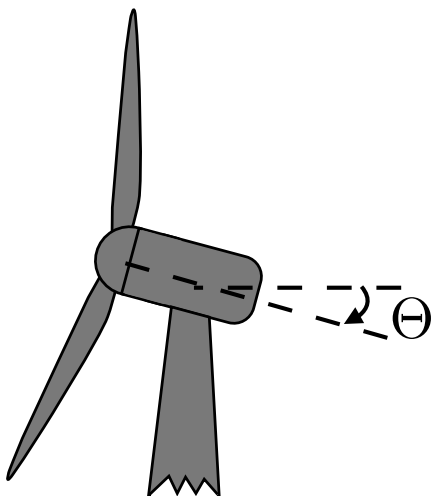

tilt

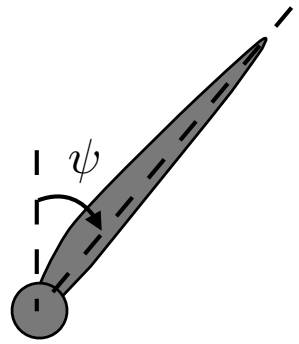

azimuth

Figure 3: Definitions for some of the relevant geometric angles.

Often this is the only modification that is applied in basic implementations, but additional corrections can also be made to the momentum side of the formulation. In developing autogyro theory, Glauert proposed a simple nonuniform inflow model to better match experimental observations [3]. The induced velocity should decrease toward the leading edge and increase toward the trailing edge. He proposed a simple linear radial variation with a $1 \mathrm{P}$ harmonic variation

$$
a_{\text {yaw }}=a\left(1+K(\chi) \frac{r}{R} \sin \psi\right)
$$

where $K$ is some unspecified function, generally expressed in terms of the wake-skew angle. The Pitt and Peters model (static, thrust effect only) was shown to agree well with experimental data as compared to several other simple inflow models [13]. Using the Pitt and Peters model leads to a formulation

$$
a_{\text {yaw }}=a\left[1+\frac{15 \pi}{64} \tan \frac{\chi}{2} \frac{r}{R} \sin \psi\right]
$$

The wake-skew angle can be estimated approximately using the relationship from Burton [14]

$$
\chi=(0.6 a+1) \chi_{0}
$$

where $\chi_{0}$ is the angle between the vector normal to the rotor plane and the wind vector (e.g., the yaw angle in the case of no tilt).

The coefficient used in Eq. (30) differs from that used in the previous version of AeroDyn. This implementation uses $15 \pi / 64$ as opposed to the factor $15 \pi / 32$ that was used previously. Comparison studies by Snel and Schepers [9] explored several different yaw models and found a Pitt/Peters model with a coefficient of $15 \pi / 64$ to fit best. The same study also uses a a nonlinear model from Delft University of Technology that uses a curve fitting approach based on a vortex ring model. They found that this model exaggerated the amplitude of the correction and removed the nonlinear terms, resulting in a model identical to Pitt/Peters but with a coefficient of 1 . The original Pitt papers [7] suggest that a denominator of 64 is appropriate for correcting thrust. Burton [14] shows a derivation relating the thrust correction to the axial induction factor 
that results in a denominator of 32 , but that result only holds for small axial inductions. In our comparisons to experimental data, we also found that the smaller factor, $15 \pi / 64$, resulted in a better fit. In addition, for coefficients larger than 1, a small minority of cases would cause the induction factor to become larger than 1 creating singularities in the solution space. Using a coefficient smaller than 1 , removed those instabilities.

Many other momentum correction methods exist, but for all, the solution procedure is exactly the same as the non-yawed case. The only modification is that $a_{y a w}$ is used in Eq. (22) instead of $a$.

\section{Coupled Method}

The correction methods of Section III are simplifications in that the inclusion of a skewed wake only directly affects the axial component of the velocity. In reality, the momentum in both the axial and tangential directions is affected. The coupled formulation described by Burton [14] is extended here. For this formulation, the two BEM equations cannot be reduced to one in the same way they are in the previous section because the axial and tangential induction factors are coupled. The coupling leads to a complicated equation for the magnitude of the inflow velocity

$$
\begin{aligned}
W^{2} & =\left[V_{x}^{\prime}\left(\cos \chi_{0}-a\right)+V_{y}^{\prime} a^{\prime} \sin \chi \cos \psi(1+\sin \chi \sin \psi)\right]^{2} \\
& +\left[V_{y}^{\prime}\left(1+a^{\prime} \cos \chi(1+\sin \chi \sin \psi)\right)+V_{x}^{\prime} \cos \psi\left(a \tan \frac{\chi}{2}-\sin \chi_{0}\right)\right]^{2}
\end{aligned}
$$

where $\chi$ is computed in the same manner as noted in Eq. (31). The velocities in this expression $\left(V_{x}^{\prime}, V_{y}^{\prime}\right)$ should include all components of velocity (shear, turbulence, structural vibration), but should not include any coordinate transformations to the local blade coordinate system with the exception of prebend. In other words, for a straight preconed blade $V_{x}^{\prime}=\left(V_{\infty}+v_{x}\right) \cos \Phi$ and $V_{y}^{\prime}=\Omega r \cos \Phi+v_{y}$ where the lowercase velocities $(v)$ contain all other external velocity contributions. Note that while the y-direction in $V_{y}^{\prime}$ is the same as used previously, the direction of $V_{x}^{\prime}$ is not the same as before but is rather in the freestream direction. The effects of skew are already included in these equations and should not be double counted by including skew in velocity coordinate transformations.

With the inflow velocity, the blade element expression for thrust coefficient is the familiar one, while the angular momentum is complicated slightly by yaw

$$
\begin{aligned}
C_{\text {Telement }} & =\left(\frac{W}{V_{\infty}}\right)^{2} \sigma^{\prime} c_{x} \\
C_{Q_{\text {element }}} & =\left(\frac{W}{V_{\infty}}\right)^{2} \sigma^{\prime}\left(c_{y} \cos \chi-c_{x} \sin \chi \cos \psi\right)
\end{aligned}
$$

For momentum theory there are various options. The momentum region only applies for $a<\beta$ where $\beta$ is typically between 0.3 and 0.5 [11]. In AeroDyn, $\beta$ is implemented as 0.4 by default. In the momentum region, Glauert's theory leads to

$$
C_{\text {Tmomentum }}=4 a F \sqrt{1-a\left(2 \cos \chi_{0}-a\right)}, \quad \text { for } a \leq \beta
$$

Vortex theory leads to an alternative form of the momentum equation.

$$
C_{\text {Tmomentum }}=4 a F\left(\cos \chi_{0}+\tan \frac{\chi}{2} \sin \chi_{0}-a \sec ^{2} \frac{\chi}{2}\right), \quad \text { for } a \leq \beta
$$

This equation from vortex theory led to results that were very similar to those of Glauert's theory, but was found to not converge as reliably at very high skew angles. For that reason, only Glauert's theory (Eq. (34)) is currently implemented in AeroDyn. For the propeller brake region $a>1$, we use the standard formula but multiply by the cosine of the inflow skew angle. This region is of lesser importance because it rarely occurs for wind turbines

$$
C_{\text {Tpropeller-brake }}=4 a F(a-1) \cos \chi_{0}, \quad \text { for } a>1
$$

The final region is the empirical region for $\beta<a<1$. Like Buhl's correction method used in the uncoupled solution [11], we assume that the thrust coefficient varies quadratically between $a=\beta$ and $a=1$. This yields a quadratic equation with three unknowns, and three boundary conditions: the thrust coefficient must equal 
that of the momentum method at $a=\beta$, the derivative $d C_{T} / d a$ must equal that of the momentum method at $a=\beta$, and the function value must be specified at $a=1$. Typically this function value is given as 2.0, but for skewed angles we multiply by the cosine of the inflow skew angle: $C_{T}(a=1) \equiv f_{1}=2 \cos \chi_{0}$. With the boundary conditions specified, the thrust coefficient in the empirical region can be solved as:

$$
C_{\text {Tempirical }}=k_{0}+k_{1} a+k_{2} a^{2}, \quad \text { for } \beta<a<1
$$

where

$$
\begin{aligned}
& f_{0}=\left.C_{\text {Tmomentum }}\right|_{a=\beta} \\
& f_{0}^{\prime}=\left.\frac{d C_{\text {Tmomentum }}}{d a}\right|_{a=\beta} \\
& f_{1}=2 \cos \chi_{0} \\
& k_{2}=\frac{f_{1}-f_{0}-f_{0}^{\prime}(1-\beta)}{(1-\beta)^{2}} \\
& k_{1}=f_{0}^{\prime}-2 k_{2} \beta \\
& k_{0}=f_{1}-k_{1}-k_{2}
\end{aligned}
$$

The angular momentum equation is

$$
C_{Q_{\text {momentum }}}=4 \frac{V_{y}^{\prime}}{V_{x}^{\prime}} a^{\prime} F\left(\cos \chi_{0}-a\right)\left(\cos ^{2} \psi+\cos ^{2} \chi \sin ^{2} \psi\right)
$$

Equating the force coefficients from linear and angular momentum gives two residual equations

$$
\begin{aligned}
& \mathcal{R}_{1}\left(a, a^{\prime}\right)=C_{\text {Telement }}-C_{\text {Tmomentum }} \\
& \mathcal{R}_{2}\left(a, a^{\prime}\right)=C_{Q_{\text {element }}}-C_{Q_{\text {momentum }}}
\end{aligned}
$$

Depending on the region that $a$ falls into, $C_{T \text { momentum }}$ in the above expression would be replaced by $C_{\text {Tempirical }}$ or $C_{\text {Tpropeller-brake }}$.

The momentum theory is derived on the basis of the entire rotor disc. However, like the classical BEM theory, it is generally applied on an annular ring. In that case, the forces and moments should be integrated around the azimuth. In the previous equations, the azimuthal integration was ignored for simplicity, and the equations were applied directly at a given azimuth. An azimuthal average value of the thrust and torque coefficient for the blade element portion is given by

$$
\begin{aligned}
& C_{\text {Telement }}=\frac{\sigma^{\prime}}{2 \pi} \int_{0}^{2 \pi}\left(\frac{W}{V_{\infty}}\right)^{2} c_{x} d \psi \\
& C_{Q_{\text {element }}}=\frac{\sigma^{\prime}}{2 \pi} \int_{0}^{2 \pi}\left(\frac{W}{V_{\infty}}\right)^{2}\left(c_{y} \cos \chi-c_{x} \sin \chi \cos \psi\right) d \psi
\end{aligned}
$$

For momentum theory, the thrust component has no azimuthal variation, and so its equation is unchanged while the torque coefficient can be integrated analytically as

$$
C_{Q_{\text {momentum }}}=2 \frac{V_{y}^{\prime}}{V_{x}^{\prime}} a^{\prime} F(\cos \gamma-a)\left(1+\cos ^{2} \chi\right)
$$

Instead of recomputing the force coefficients along the azimuth at each time step, we save the time history of the force coefficients from the past revolution to use in the integration. Thus, we are using a running azimuthal average. This integral approach is not yet implemented in AeroDyn and so comparisons in this paper use the instantaneous azimuthal version (Eq. (33)).

The solution approach must be modified because there are now 2 state variables at each radial section ( $a$ and $a^{\prime}$ ). Brent's method can no longer be used, and an n-dimensional root-finding algorithm is necessary. AeroDyn uses a Powell-hybrid method to solve the residual equations. These equations converge most of the time, but cannot be guaranteed to converge like the uncoupled method can. In cases where the solution fails, both induction factors are set to 0 . This generally only occurs at very large skew angles. Once the induction factors are found, the distributed loads are computed in the same way (Eqs. (23)-(27)). 


\section{Implementation and Changes in AeroDyn}

The latest version of AeroDyn (v15) is being overhauled to adhere to the modularization framework [2]. AeroDyn provides the aerodynamics modeling capabilities for NREL's FAST wind turbine aero-hydro-servoelastic simulation tool (Fig. 4a). The BEMT sub-module of AeroDyn v15 (standing for BEM theory) implements the BEM equations and solution algorithm (Fig. 4b). In this process, the code has been revised, and new theoretical approaches have been implemented or are in the process of being integrated within the software. The BEMT and Generalized Dynamic Wake (GDW) methods are independent sub-modules of AeroDyn. As with other AeroDyn sub-modules, such as Unsteady Aero, they can be enabled through input file settings. The connections shown in Fig. 4b do not imply actual linkages within the code. Here we focus on the most relevant differences between AeroDyn v15 and v14 as they relate to the BEMT sub-module, and implementation details pertinent to the topic discussed in this study.

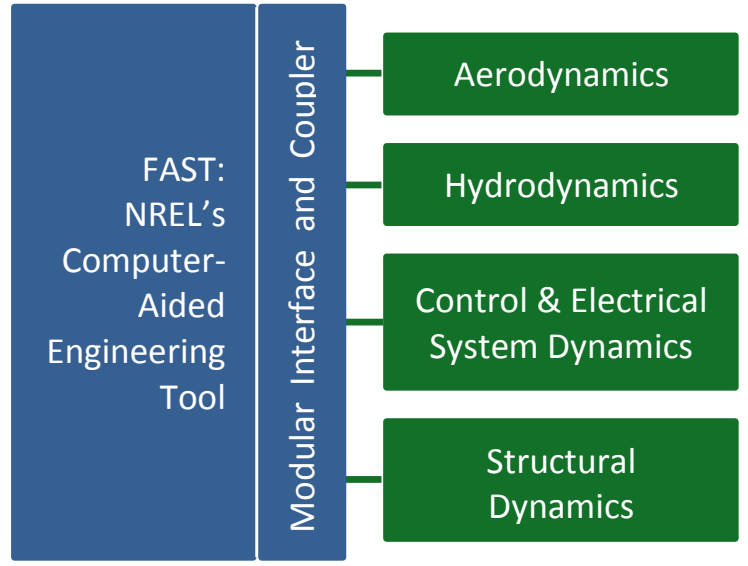

(a) Conceptual diagram of FAST and its modular interface.

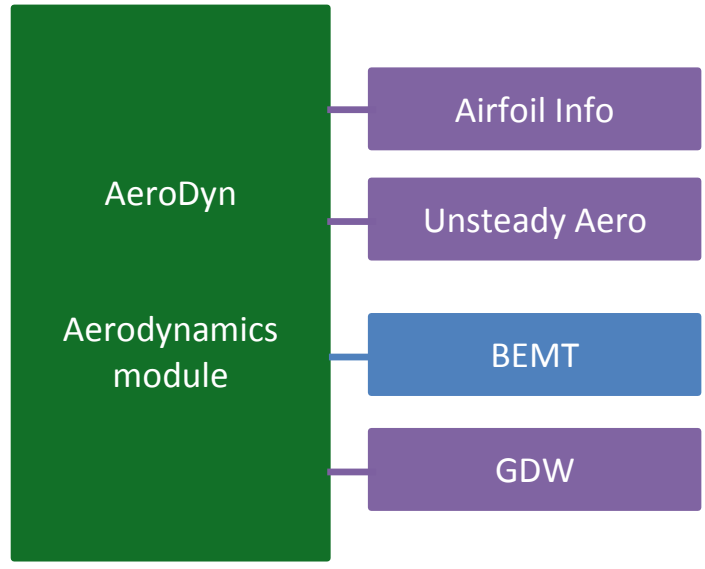

(b) The BEMT module is a sub-module within AeroDyn.

Figure 4: AeroDyn is a module within NREL's FAST, an aero-hydro-servo-elastic simulation tool. The focus of this study was on AeroDyn's BEMT sub-module.

\section{A. Implementation in FAST Framework}

Following the requirements of the modularization framework, we establish the inputs, outputs, states, and parameters that allow the BEM equations to be properly coupled to AeroDyn (and in turn to FAST). The nomenclature section identifies each variable by type; the variables without a specific type identified are variables local to a given routine. Within the framework, static quantities are specified during the module's initialization and are stored as parameters. For the BEMT module, these parameters include the undeflected rotor geometry, air density, kinematic viscosity, airfoil properties associated with each blade node, and BEMT algorithm options. These options include selection of the skewed-wake correction method, use of $\operatorname{drag}\left(C_{d}\right)$ when computing the induction factors (drag is always used for load calculations), application of the Prandtl hub/tip loss corrections, and the inclusion of the tangential induction factor $\left(a^{\prime}\right)$ in the solution. Disabling the latter option is achieved by simply setting $a^{\prime}$ to zero in Eq. (21). The algorithm of Section III includes the ability to iterate on Reynolds number. However, Aerodyn v15 does not implement this additional iteration loop.

During time-marching, AeroDyn provides BEMT with the required time-dependent inputs relating to the blade node positions and displacements. These inputs are specified for the entire rotor, for a given blade, or for a specific blade node location. The rotor-related inputs are angular velocity $(\Omega)$ and inflow skew angle $\left(\chi_{0}\right)$. For each blade, AeroDyn specifies the azimuthal position $(\psi)$. The following inputs are required at each blade node: the local twist angle $(\theta)$, the local inflow velocities $\left(V_{x}\right.$ and $\left.V_{y}\right)$, and the node's radial distance from the center-of-rotation. The latter accounts for blade deformations.

The required state variables depend on whether the user selected an uncoupled skewed wake correction from Section III, or a coupled correction from Section IV. For the uncoupled corrections, the state variable 
is the inflow angle $(\phi)$ at each blade node. For coupled skewed-wake corrections, the state variables become the axial and tangential induction factors $\left(a, a^{\prime}\right)$. These states are of the constraint (algebraic) type.

The AeroDyn module uses the BEMT outputs of $W, \phi, c_{x}$, and $c_{y}$ to compute the loads of Eqs. (25)-(27). The BEMT sub-module implements the solution algorithms described in Section II-Section IV using three key interface routines of the FAST modularization framework: CalcConstrStateResidual, UpdateStates, and CalcOutput (Fig. 5). The included flow charts only document the uncoupled BEM solution. For a given time, $t+\Delta t$, the subroutine UpdateStates determines the new optimal value of $\phi(t+\Delta t)$. If however, the previous value of $\phi$, at time $t$ satisfies the residual equation within a tolerance $(t o l)$, the work of Algorithm 1 is skipped and we set $\phi(t+\Delta t)=\phi(t)$. The CalcConstrStateResidual subroutine is used within Algorithm 1 to determine $\mathcal{R}(\phi)$. The full set of Eqs. (1)-(23), must be processed by the CalcOutput subroutine because while the states have not changed, the inputs may have changed since the previous call to UpdateStates.

Since AeroDyn v15 accommodates curved and flexible blades, some choices were made when implementing the $r$ and $R$ quantities shown in this paper. For tip and hub loss equations, $r, R_{h u b}$, and $R$ are computed as distances along the unloaded blade. These are computed only once, during initialization. However, the local solidity $\sigma^{\prime}$ is a function of $r$ which is taken as the instantaneous radial distance from the center-of-rotation to the possibly deflected blade node. The values of $r$ and $R$ in Eq. (30) are computed using the same convention.

\section{B. Primary Changes in New Version of AeroDyn}

AeroDyn v15 adopts a restructured handling of the airfoil data via the Airfoil Info sub-module. The $C_{l^{-}} C_{d^{-}} C_{m}$ airfoil coefficient data tables, which are a function of angle of attack, are now interpolated via cubic-splines, whereas previously, a linear interpolation was used. The airfoil coefficients may also depend on Reynolds number and aerodynamic-control setting (e.g., flap setting). Their is no dependence on these additional parameters within the BEM solution loop and so linear linear interpolation was used. However, the new scheme is based on logarithmic values of the Reynolds number for improved accuracy in the interpolation. Corrections to 2D airfoil data to account for rotational augmentation effects and delayed stall effects are currently handled through a preprocessing step (AirfoilPrep), but in the future these corrections will be implemented directly within AeroDyn.

When calculating the induction factor, AeroDyn release v14 had a simple iteration loop where the convergence was based on the difference between two successive iteration values of the axial induction factor alone. The loop also made use of a relaxation factor to assign the new step to the induction factor. The tangential induction factor was decoupled and calculated at each iteration based on the axial induction. AeroDyn v14 only models straight blades. The body motion contribution to the axial velocity was not corrected for induction, and no elastic-body motion contribution was considered in the calculation of the effective in-plane velocity (the latter is considered an unintentional bug). Within the tangential induction calculation, the critical phi values of $0^{\circ}$ and $90^{\circ}$ were avoided, limiting the cosine and sine of the inflow angle to 0.01 .

AeroDyn v15 adopts a new solution method as described in Section III, where $a$ and $a^{\prime}$ are solved simultaneously. Additionally, the body motion is added to the wind in- and out-of-plane components. This seems more appropriate from a pure aerodynamics stand-point, especially for larger wind turbines where turbulence and structural dynamics time scales may be closer to each other.

Another important difference between the two versions of AeroDyn is that the skewed wake correction (e.g., Pitt-Peters) is implemented within the iterator in the new implementation as opposed to a-posteriori in v14. AeroDyn v14 loosely implemented the Pitt-Peters method by including a factor dependent on the disk-averaged angle of attack $\left(\alpha_{d}\right)$ and a correction factor of $15 \pi / 32$. AeroDyn v15 uses the skew angle $(\chi)$ in place of $\alpha_{d}$ and a correction factor of $15 \pi / 64$. 


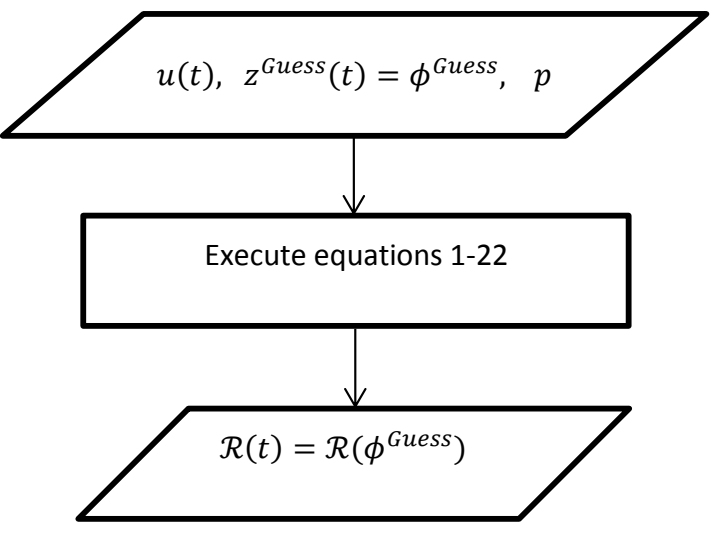

(a) The CalcConstrStateResidual subroutine.

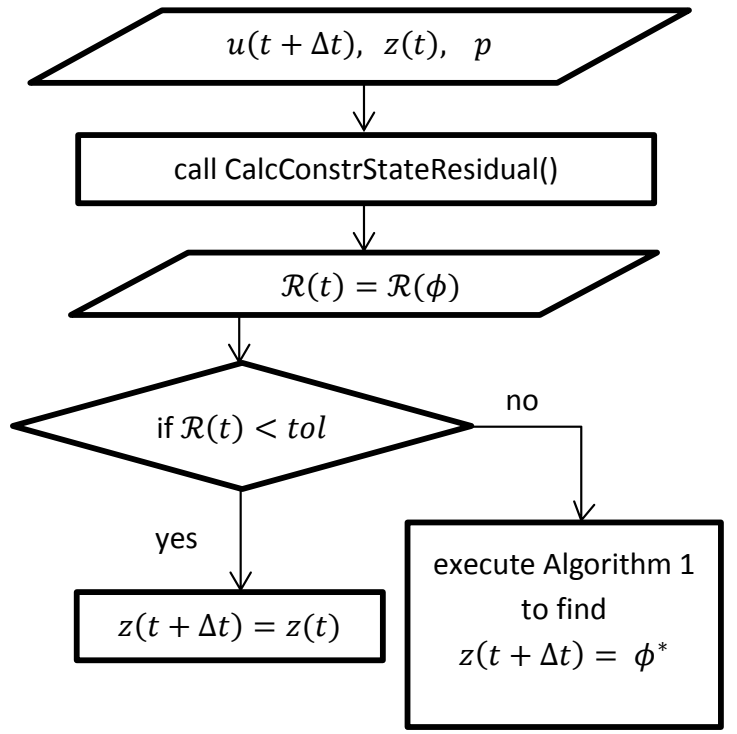

(b) The UpdateStates subroutine.

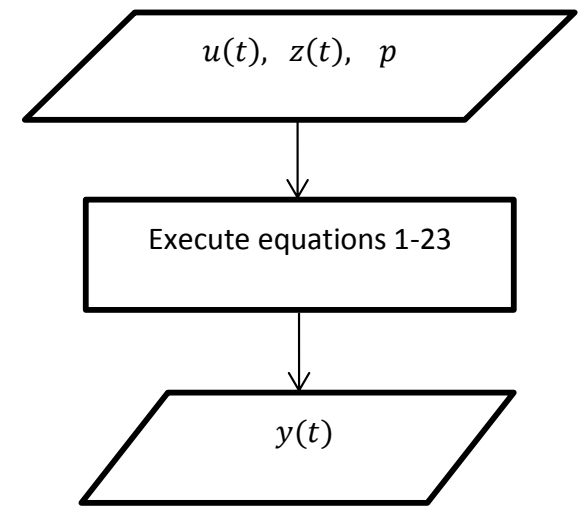

(c) The CalcOutput subroutine.

Figure 5: The uncoupled BEM solution technique as implemented in the BEMT sub-module via the FAST modularization framework. 


\section{Comparison to Experimental Data}

In this section, we compare the simulation results from AeroDyn v15 and v14 to the data from the NREL Unsteady Aerodynamics Experiment (UAE) [15]. The UAE tests were conducted at the NASA $80 \mathrm{x}$ 120 foot wind tunnel with a $20-\mathrm{kW}$ horizontal-axis wind turbine. A large variety of tests were conducted, but this paper focuses on comparison with the "upwind baseline" test series (Sequence H). All input values were either taken directly from the report or from the experimental data files. For the S809 airfoil data, the measurements were taken from the Ohio State University (OSU) wind tunnel at a Reynolds number of 750,000. The FoilCheck utility (a precursor to AirfoilPrep) was used to include corrections/extensions of the data at seven radial stations to account for the effects of deep stall and rotational stall delay based on a blade aspect ratio of 11; stall delay was not applied outboard of $41 \%$ span. Additionally, a cylindrical section was assumed at the blade root. These same airfoil data have been used in other projects involving analysis of the UAE datasets [16].

A small shear exponent of 0.05 was used, as was consistent with the experimental data. Distributed loads were reported in the UAE dataset using an integration of pressure from data gathered at pressure taps. The results reported here are modified only in that the loads were rotated back to the blade coordinate system rather than reported in the rotor coordinate system (dividing by the cosine of the precone angle). At a given azimuth angle, some measured inputs show small variations during the 30-second measurements, and average values were used for each azimuthal location. Distributed loads displayed at a fixed azimuth angle use all experimental data points within $\pm 1^{\circ}$ in azimuth. For results displayed as a function of azimuth angle, all the data points from the 30-second interval are shown. In all of the figures of this section, circles are used to signify experimental data, whereas continuous lines represent simulation results. Distributed loads in the $\mathrm{x}$-direction are referred to as flapwise, while loads in the y-direction are referred to as lead-lag.

The first comparison is done at zero yaw and is a comparison between basic BEM theory and the experimental data. Figures 6 and 7 compare the lead-lag and flapwise loads along the blade at three different wind speeds. Each case is done at $0^{\circ}$ yaw and $90^{\circ}$ azimuth. Because the shear is so small and the yaw angle is zero, the computational and simulation results differ only slightly as a function of azimuth angle. The BEM model generally shows good agreement to the experimental data except at the inboard sections for high wind speeds. These locations are operating post-stall where experimental airfoil data was lacking and where the rotational augmentation effects (stall delay) are not completely accurate in our models. The purpose of this study is to isolate the effect of the yaw models, and so we specifically restrict our focus to low wind speeds where different stall models (or lack thereof) will not confound the results. Pre-stall operation is also of most relevance to the analysis of modern utility-scale blades with full-span pitch control during normal operation. Unsteady aerodynamics and dynamic stall models are under development and were not considered in this work.

For the remainder of the section, results are shown at a constant wind speed of $V_{\infty}=7 \mathrm{~m} / \mathrm{s}$. At the lower wind speeds and outboard at $10 \mathrm{~m} / \mathrm{s}$, we note that the lead-lag loads are predicted quite well. This is important because those are the loads that affect power prediction. The flapwise loads lead to the thrust force and are well predicted inboard but appear to be overpredicted toward the blade tips.

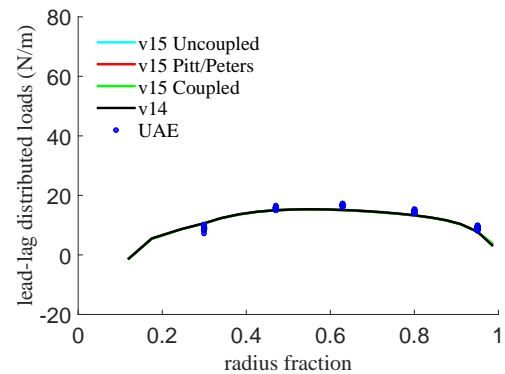

(a) $5 \mathrm{~m} / \mathrm{s}$

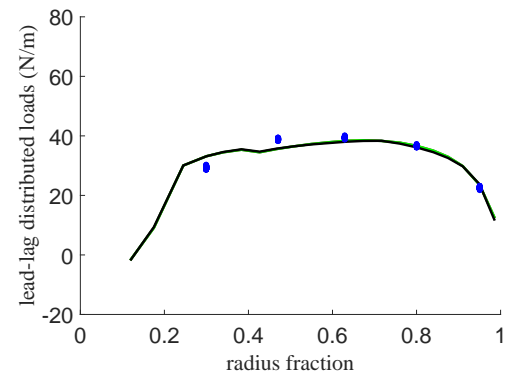

(b) $7 \mathrm{~m} / \mathrm{s}$

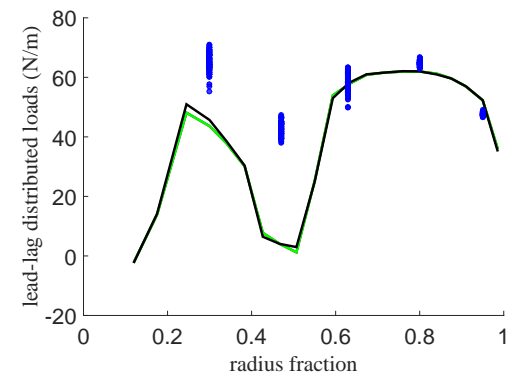

(c) $10 \mathrm{~m} / \mathrm{s}$

Figure 6: Lead-lag loads along the blade at three different wind speeds with zero yaw and a fixed azimuth $\left(\gamma=0^{\circ}, \psi=90^{\circ}\right)$. The circles correspond to experimental data and the lines to simulation results.

Figures 8-11 show the blade loading at six different yaw angles for two different azimuth angles $\left(90^{\circ}\right.$ 


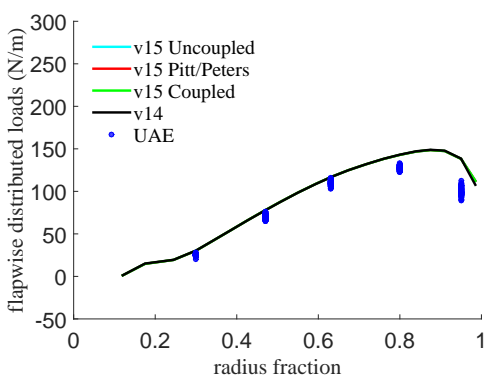

(a) $5 \mathrm{~m} / \mathrm{s}$

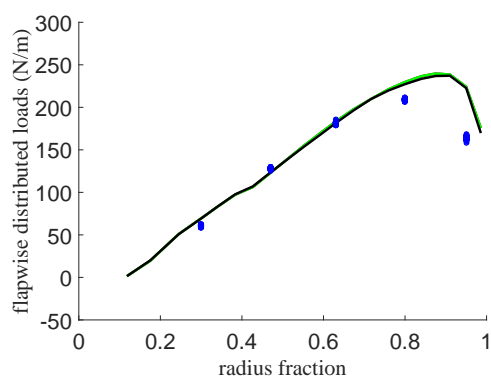

(b) $7 \mathrm{~m} / \mathrm{s}$

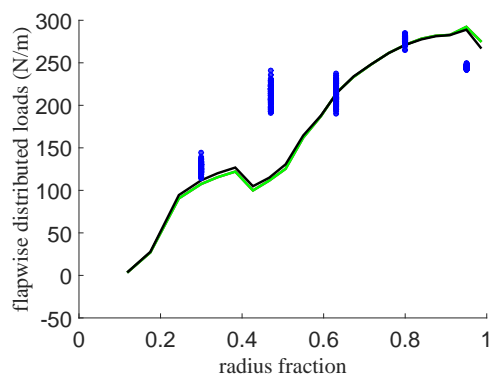

(c) $10 \mathrm{~m} / \mathrm{s}$

Figure 7: Flapwise loads along the blade at three different wind speeds with zero yaw and a fixed azimuth $\left(\gamma=0^{\circ}, \psi=90^{\circ}\right)$. The circles correspond to experimental data and the lines to simulation results.

azimuth in Figs. 8 and 9 and $270^{\circ}$ azimuth in Figs. 10 and 11). In each plot, three different analysis methods are used for AeroDyn v15, and comparisons are also shown for AeroDyn v14. The first method with v15 does not apply any corrections; it does make use of the local velocity computation (Eq. (28)), which is a function of yaw and azimuth. The second method is the Pitt/Peters model (Eq. (30)). At yaw angles of $0^{\circ}$ and $180^{\circ}$, these two methods are identical. The third method is a coupled method as described in Section IV.

We note that even with no correction (other than the local velocity calculation), the BEM model does reasonably well at predicting the load distribution across a wide range of yaw angles. While the Pitt/Peters does improve the flapwise loading, particularly at large yaw angles, using no correction gives better results for the lead-lag loads. The improvement in flapwise loads is not surprising as the methodology was designed as a thrust correction, but the decrease in accuracy of the lead-lag loads may be an unacceptable trade-off, especially because those loads are responsible for the prediction on power production. Of those two methods, using no correction may be a better alternative for large yaw angles.

The coupled solution is the most accurate, especially for the lead-lag loads. The flapwise loading for the coupled solution tends to be overpredicted toward the blade tips (for some azimuth angles), but the accurate lead-lag loads should allow for superior predictions for power generation. In almost all cases, any of the AeroDyn v15 methods are more accurate than the methodology of v14.

Figures 12 and 13 show the variation in flapwise and lead-lag loads as a function of azimuth angle at three different radial stations along the blade. Unsteady airfoil aerodynamic effects are not included. Each plot is done at a fixed wind speed of $7 \mathrm{~m} / \mathrm{s}$ and a fixed yaw angle of $40^{\circ}$. The uncoupled method is symmetric about $180^{\circ}$ azimuth. The shape of the uncorrected method is perhaps the least accurate, but because the peaks in its curve are also the most modest, it tends to never stray too far from the experimental results. Unlike the uncorrected method, we see that the Pitt/Peters model is able to capture asymmetry in the loading across the azimuth variation. The Pitt/Peters model predicts a decrease in loading on the downwind side (positive yaw, $90^{\circ}$ azimuth), and conversely an increase in loading for the upwind side at $270^{\circ}$ azimuth. However, the assumed sinusoidal variation in the Pitt/Peters correction often overpredicts the amplitude of the variation, particularly towards the blade tips, and a sinusoidal variation appears to be a poor model for some conditions. The curve for the coupled solution method, while not perfectly matched in magnitude, shows remarkably good agreement in shape. The coupled solution method again displays higher accuracy as compared to the other methods. The AeroDyn v14 method uses the Pitt/Peters method but with a larger correction factor. As the v15 Pitt/Peters correction already overpredicts the variation, unsurprisingly the v14 methodology overpredicts by an even larger amount. 


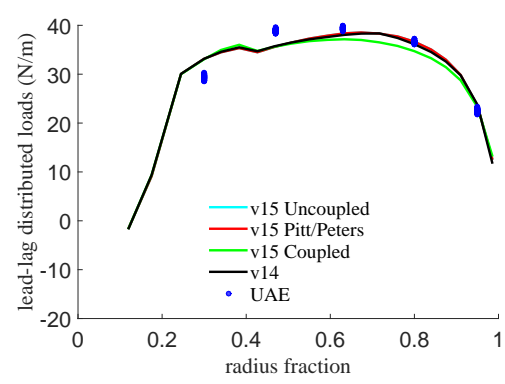

(a) $\gamma=0^{\circ}$

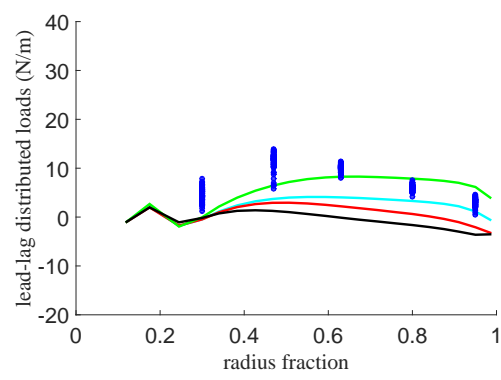

(d) $\gamma=60^{\circ}$

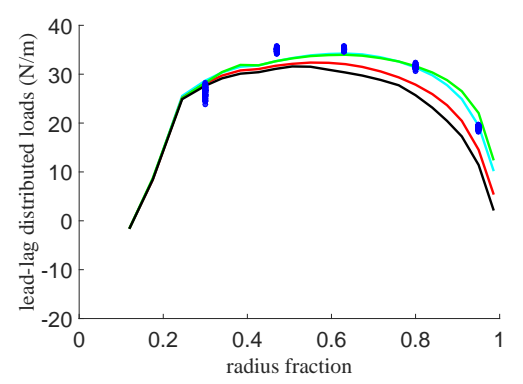

(b) $\gamma=20^{\circ}$

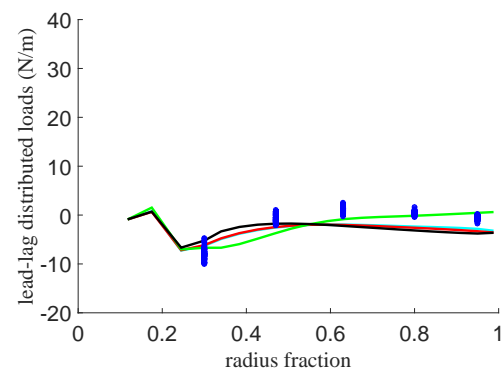

(e) $\gamma=75^{\circ}$

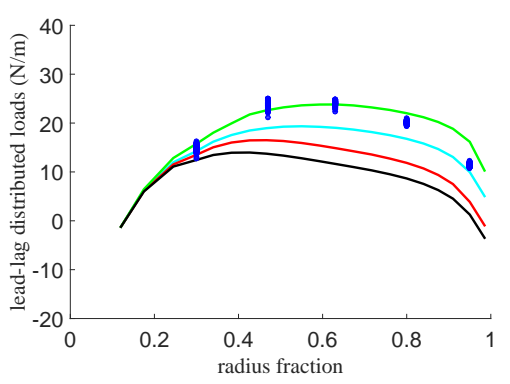

(c) $\gamma=40^{\circ}$

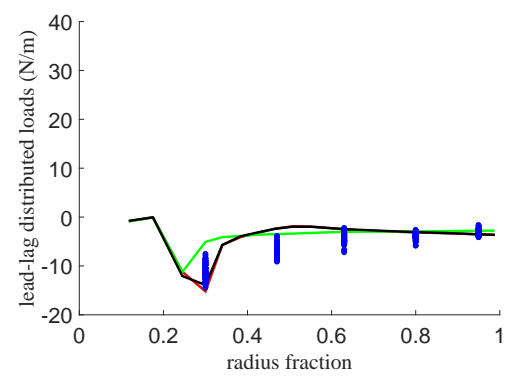

(f) $\gamma=90^{\circ}$

Figure 8: Distributed lead-lag loads along blade at six different yaw angles $\left(V_{\infty}=7 \mathrm{~m} / \mathrm{s}, \psi=90^{\circ}\right)$.

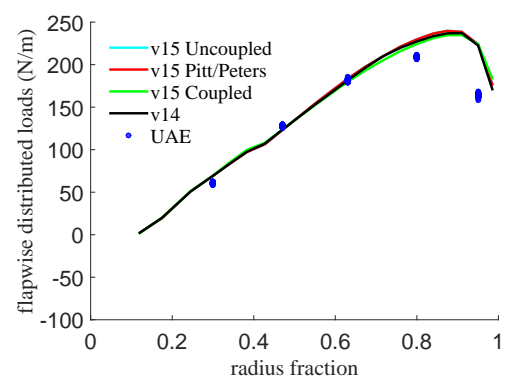

(a) $\gamma=0^{\circ}$

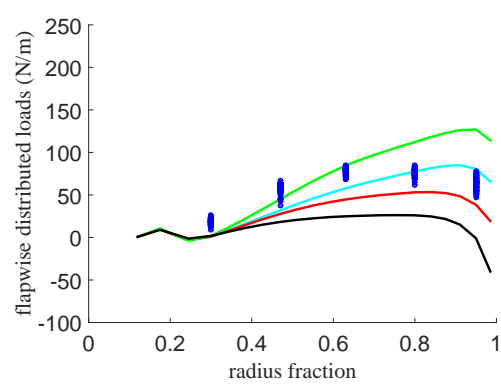

(d) $\gamma=60^{\circ}$

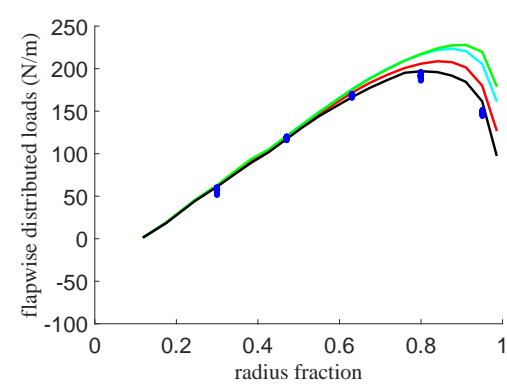

(b) $\gamma=20^{\circ}$

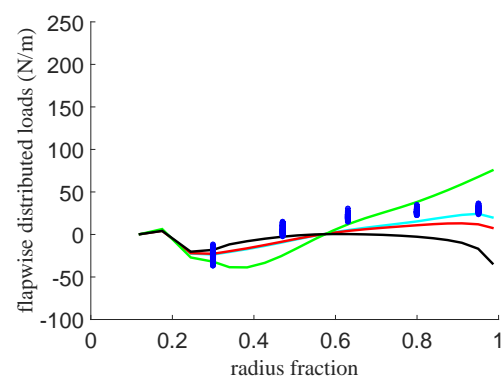

(e) $\gamma=75^{\circ}$

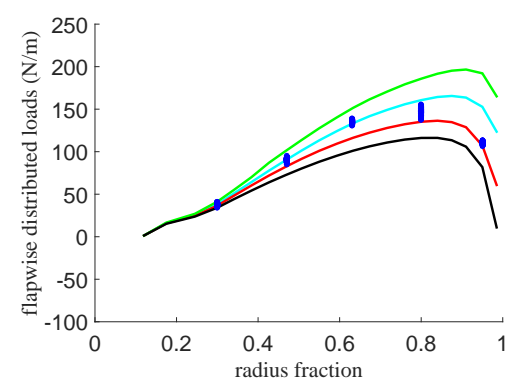

(c) $\gamma=40^{\circ}$

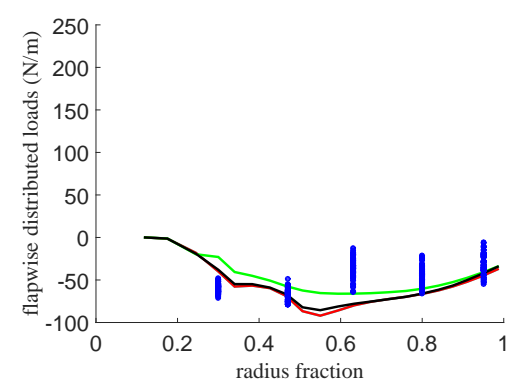

(f) $\gamma=90^{\circ}$

Figure 9: Distributed flapwise loads along blade at six different yaw angles $\left(V_{\infty}=7 \mathrm{~m} / \mathrm{s}, \psi=90^{\circ}\right)$. 


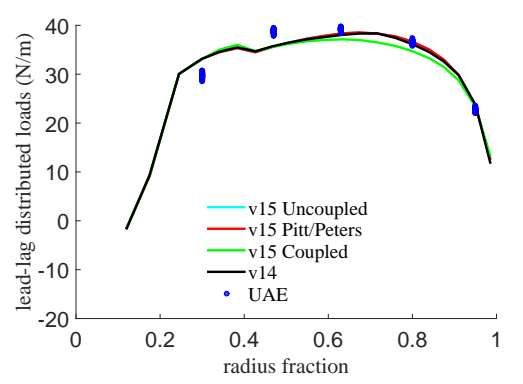

(a) $\gamma=0^{\circ}$

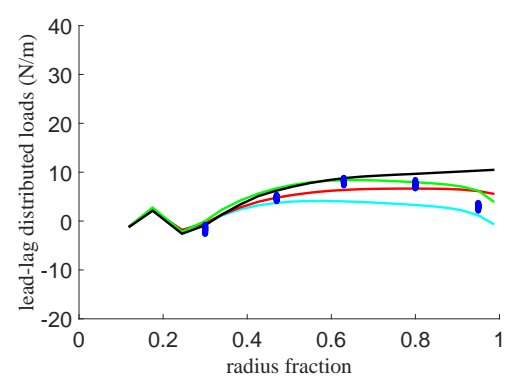

(d) $\gamma=60^{\circ}$

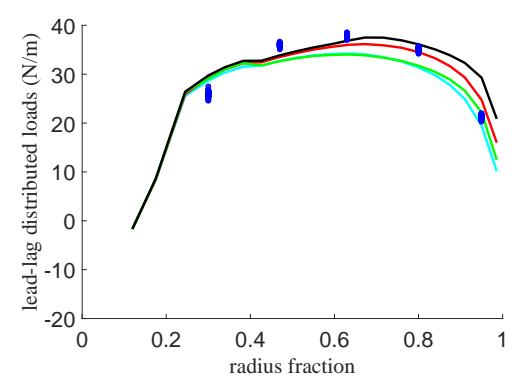

(b) $\gamma=20^{\circ}$

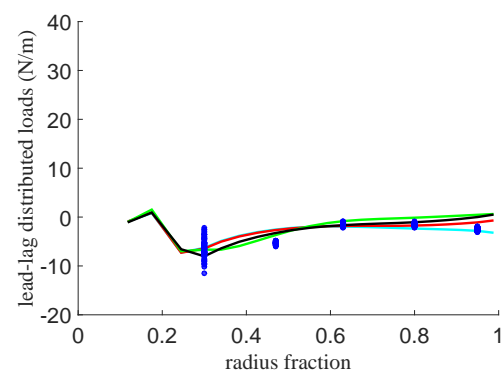

(e) $\gamma=75^{\circ}$

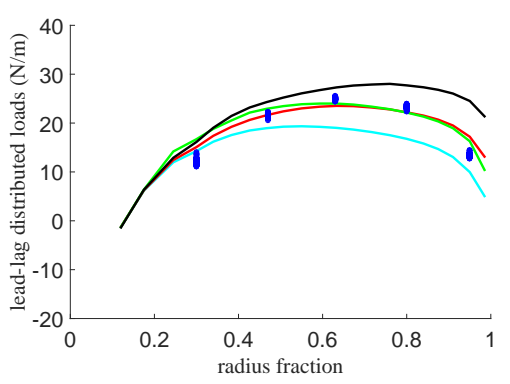

(c) $\gamma=40^{\circ}$

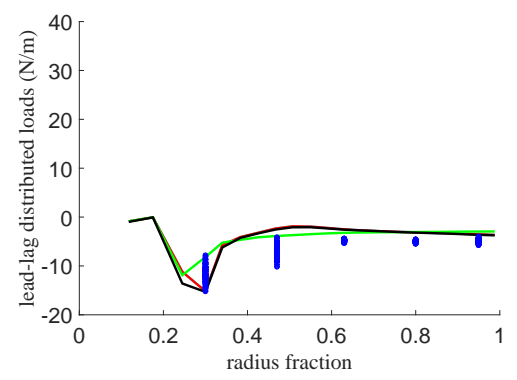

(f) $\gamma=90^{\circ}$

Figure 10: Distributed lead-lag loads along blade at six different yaw angles $\left(V_{\infty}=7 \mathrm{~m} / \mathrm{s}, \psi=270^{\circ}\right)$.

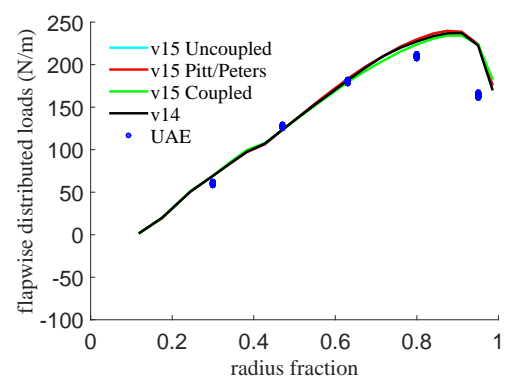

(a) $\gamma=0^{\circ}$

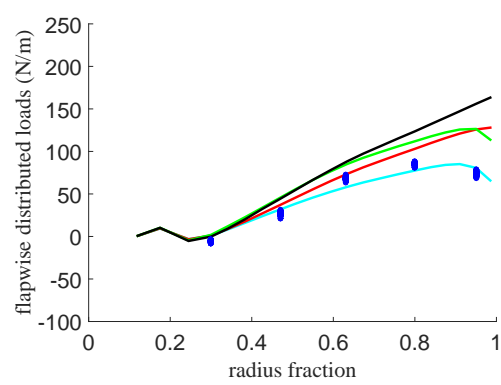

(d) $\gamma=60^{\circ}$

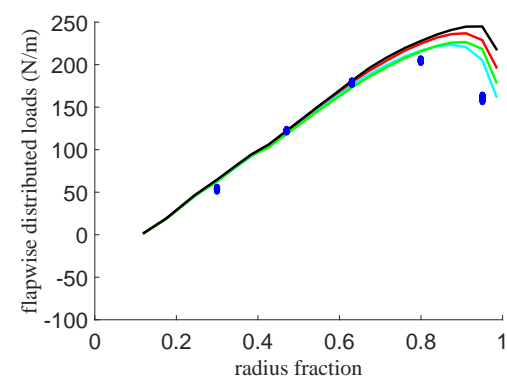

(b) $\gamma=20^{\circ}$

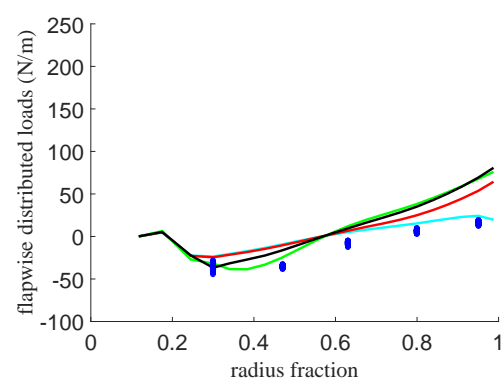

(e) $\gamma=75^{\circ}$

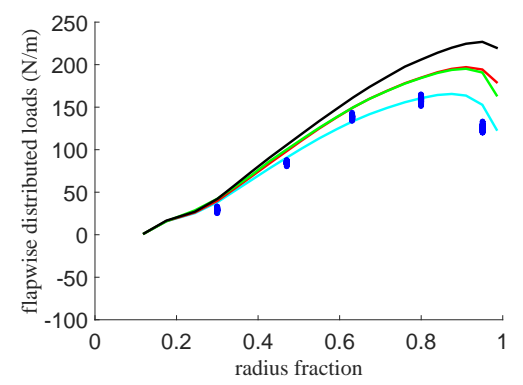

(c) $\gamma=40^{\circ}$

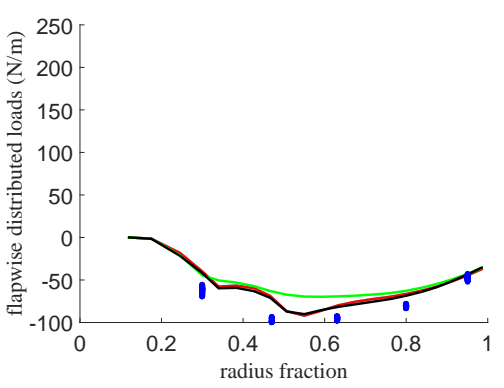

(f) $\gamma=90^{\circ}$

Figure 11: Distributed flapwise loads along blade at six different yaw angles $\left(V_{\infty}=7 \mathrm{~m} / \mathrm{s}, \psi=270^{\circ}\right)$. 


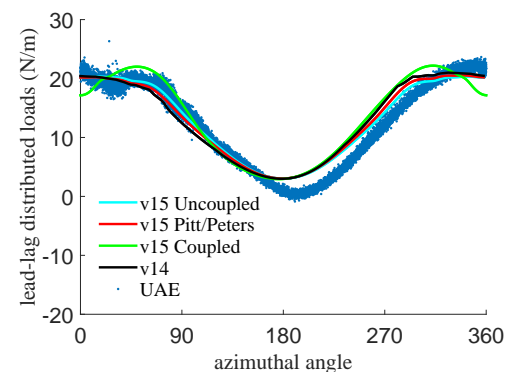

(a) $r / R=0.3$

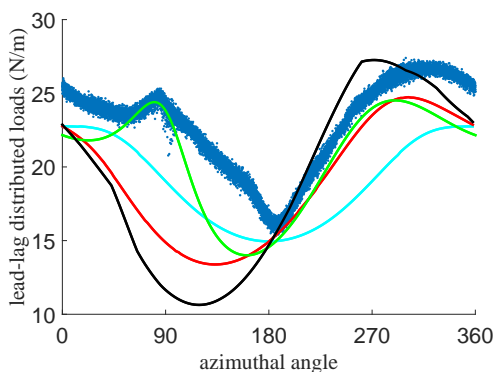

(b) $r / R=0.63$

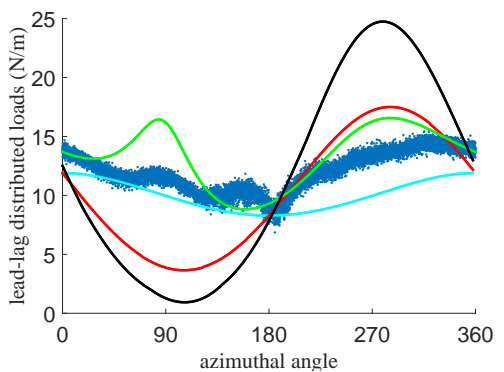

(c) $r / R=0.95$

Figure 12: Lead-lag loads at different stations along the blade as a function of azimuth $\left(V_{\infty}=7 \mathrm{~m} / \mathrm{s}, \gamma=\right.$ $\left.40^{\circ}\right)$.

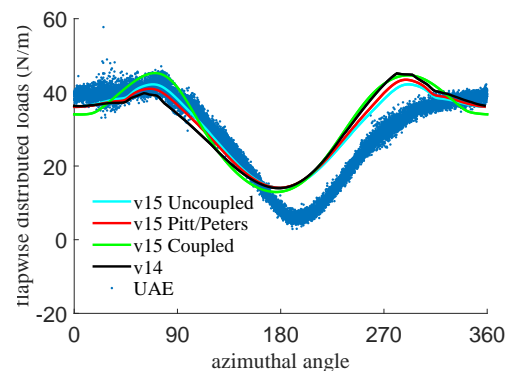

(a) $r / R=0.3$

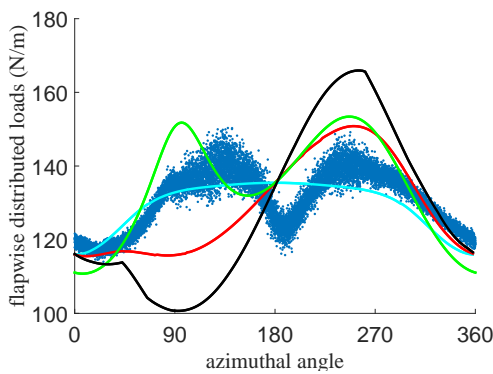

(b) $r / R=0.63$

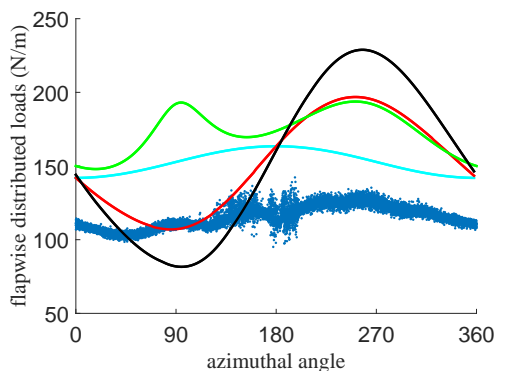

(c) $r / R=0.95$

Figure 13: Flapwise loads at different stations along the blade as a function of azimuth $\left(V_{\infty}=7 \mathrm{~m} / \mathrm{s}, \gamma=\right.$ $\left.40^{\circ}\right)$. 


\section{Conclusion}

This paper discussed updates to NREL's AeroDyn, in particular, improvements in the BEM solution algorithm and the skewed-wake modeling within the new FAST modularization framework [2]. Two classes of correction methods were introduced: the classical methods based on Glauert's theory, and the more rigorous methods where axial and tangential momentum equations are coupled.

Comparisons have been shown between the new approach in AeroDyn v15, the previous AeroDyn v14, and experimental data from the NREL Unsteady Aerodynamics experiment. At $0^{\circ}$ yaw, the two versions of AeroDyn are indistinguishable, and results follow the experimental data fairly well, especially at the inboard section of the blade. Discrepancies were noted toward the tip for the flapwise loads and, at higher wind speeds near the root where the sections are operating post-stall. It is argued that the noted differences are due to lack of experimental data post-stall, imperfect tip-loss corrections, and to stall delay associated with rotational augmentation effects near the mid-section of the blade not fully captured by the models.

At yaw angles greater than $0^{\circ}$, differences can be seen in the results provided by the two versions of AeroDyn. Both lead-lag and flapwise forces are better captured by v15. The discrepancy can be attributed to a slightly different correction implemented in v15, and in the fact that the yaw correction is integral part of the induction solver calculation in v15, whereas it was done a-posteriori in v14. It was also observed that the inclusion of the Pitt/Peters correction improved the shape of the variation across azimuth angles but did not necessarily lead to more accurate loading along the blades. Ignoring the induction calculation correction and just accounting for the velocity changes along the blades as a function of yaw, gave arguably more accurate results across a wide range of yaw angles. This is an option that can now be exercised in AeroDyn v15. The coupled solution method was significantly more accurate than either of the uncoupled solution approaches. The only limitation of the coupled solution approach is that solutions are not always guaranteed.

This study did not account for unsteady airfoil aerodynamics, including dynamic stall corrections, mainly on the basis that for limited wind speeds (high tip-speed ratios), these effects should be negligible. Future research efforts will need to incorporate these effects and validate the models with applicable experimental data. Attention should be paid to gathering post-stall data, using refined tip-loss corrections, and modeling stall delay and centrifugal pumping effects. Finally, efforts are already underway to explore other skewed wake models like the integral coupled approach, add generalized dynamic wake theory, and couple AeroDyn v15 with FAST v8.

\section{Acknowledgments}

This work was supported by the U.S. Department of Energy under Contract No. DE-AC36-08GO28308 with the National Renewable Energy Laboratory.

\section{References}

\footnotetext{
${ }^{1}$ Ning, A., "A Simple Solution Method for the Blade Element Momentum Equations with Guaranteed Convergence," Wind Energy, Vol. 17, No. 9, Sep 2014, pp. 1327-1345.

${ }^{2}$ Jonkman, J., "The New Modularization Framework for the Fast Wind Turbine Cae Tool Tool," 51st AIAA Aerospace Sciences Meeting including the New Horizons Forum and Aerospace Exposition, Dallas, TX, Jan 2013.

${ }^{3}$ Glauert, H. and Committee, A. R., A General Theory of the Autogyro, HM Stationery Office, Nov 1926.

${ }^{4}$ Coleman, R. P., Feingold, A. M., and Stempin, C. W., "Evaluation of the Induced-Velocity Field of an Idealized Helicoptor Rotor," Tech. Rep. ARR L5E10, NACA, 1945.

${ }^{5}$ Drees, J. M., "A Theory of Airflow through Rotors and Its Application to Some Helicopter Problems," Journal of the helicopter association of great britain, Vol. 3, No. 2, 1949, pp. 79-104.

${ }^{6}$ Mangler, K. and Squire, H. B., "The Induced Velocity Field of a Rotor," ARC R\&M, Vol. 2642, 1950.

${ }^{7}$ Pitt, D. M. and Peters, D. A., "Theoretical Prediction of Dynamic-Inflow Derivatives," Vertica, Vol. 5, No. 1, 1981, pp. 21-34.

${ }^{8}$ Peters, D. A., Boyd, D. D., and He, C. J., "Finite-State Induced-Flow Model for Rotors in Hover and Forward Flight," Journal of the American Helicopter Society, Vol. 34, No. 4, 1989, pp. 5.

${ }^{9}$ Snel, H., Schepers, J., and Nederland, S. E. C., "Joint Investigation of Dynamic Inflow Effects and Implementation of an Engineering Method," Tech. Rep. ECN-C-94-107, Netherlands Energy Research Foundation, 1995.

${ }^{10} \varnothing$ ye, S., "Induced Velocities for Rotors in Yaw," Proceedings of the Sixth IEA Symposium, ECN, Petten, Holland, 1992.

${ }^{11}$ Buhl, M. L., "A New Empirical Relationship between Thrust Coefficient and Induction Factor for the Turbulent Windmill State," Tech. Rep. NREL/TP-500-36834, National Renewable Energy Laboratory, Aug 2005.
} 
${ }^{12}$ Brent, R. P., "An Algorithm with Guaranteed Convergence for Finding a Zero of a Function," The Computer Journal, Vol. 14, No. 4, 1971, pp. 422-425.

${ }^{13}$ Chen, R. T. N., "A Survey of Nonuniform Inflow Models for Rotorcraft Flight Dynamics and Control Applications," Tech. Rep. NASA-TM-102219, National Aeronautics and Space Administration, Nov 1989.

${ }^{14}$ Burton, T., Jenkins, N., Sharpe, D., and Bossanyi, E., Wind Energy Handbook, Wiley, 2nd ed., 2011.

${ }^{15}$ Hand, M. M., Simms, D. A., Fingersh, L. J., Jager, D. W., Cotrell, J. R., Schreck, S., and Larwood, S. M., "Unsteady Aerodynamics Experiment Phase VI: Wind Tunnel Test Configurations and Available Data Campaigns," Tech. Rep. NREL/TP500-29955, National Renewable Energy Laboratory, Dec 2001.

${ }^{16}$ Jonkman, J. M., "Modeling of the UAE Wind Turbine for Refinement of FAST_AD," Tech. Rep. NREL/TP-500-34755, National Renewable Energy Laboratory, Dec 2003. 